\title{
Asplenium danxiaense sp. nov. (Aspleniaceae, Aspleniineae), a new tetraploid fern species from Guangdong, China, based on morphological and molecular data
}

\author{
Ke-Wang XU (1) 1, *, Chen-Xue LIN (1) 2, Jian-Qiang GUO (1) ${ }^{3}$, Xin-Xin ZHOU (1) 4, \\ Wen-Bo LIAO (1) ${ }^{5}$ \& Ling-Feng MAO (1) 6,* \\ ${ }^{1,2,6} \mathrm{Co}$-Innovation Center for Sustainable Forestry in Southern China, \\ College of Biology and the Environment, Nanjing Forestry University, \\ Nanjing, 510275, China. \\ ${ }^{3}$ Administrative Commission of Danxia mountain National Park, \\ Shaoguan 512300, Guangdong, China. \\ ${ }^{4}$ South China Botanical Garden, Chinese Academy of Sciences, \\ Guangzhou, 510650, China.
}

${ }^{5}$ State Key Laboratory of Biocontrol and Guangdong Provincial Key Laboratory of Plant Resources, School of Life Sciences, Sun Yat-sen University, Guangzhou, 510275, China.

*Corresponding authors: xukw10@njfu.edu.cn; maolingfeng2008@163.com

${ }^{2}$ Email: 1932219200@qq.com

32Email: 1368832042@qq.com

${ }^{4}$ Email: xxzhou@scbg.ac.cn

${ }^{5}$ Email: 1sslwb@mail.sysu.edu.cn

\begin{abstract}
The Asplenium coenobiale complex is distributed in Eastern Asia and Southeast Asia with its distribution center in southwestern China. In this study, we carried out a detailed morphological, cytological, and phylogenetic study by adding two samples from Danxia landform in Guangdong. The sequences of five chloroplast markers and one nuclear marker of the A. coenobiale complex were analyzed with maximum likelihood and Bayesian inference, respectively. The morphological and phylogenetic analyses support the recognition of a new species (A. danxiaense K.W.Xu sp. nov.) of the A. coenobiale complex from a cave of Danxia mountain, Guangdong province, southern China. This new species can be distinguished from $A$. coenobiale and A. pulcherrimum by having scales narrowly triangular to lanceolate, apex ending in a short apical tail, basal basiscopic pinnule usually largest, fertile segment scarce, and exospore length usually more than $50 \mu \mathrm{m}$ and shows significant molecular differences from other species in this complex. A detailed description and illustrations are presented.
\end{abstract}

Keywords. Cave, cryptic species, Danxia landform, southern China.

Xu K.W., Lin C.X., Guo J.G., Zhou X.X., Liao W.B. \& Mao L.F. 2022. Asplenium danxiaense sp. nov. (Aspleniaceae, Aspleniineae), a new tetraploid fern species from Guangdong, China, based on morphological and molecular data. European Journal of Taxonomy 798: 162-173.

https://doi.org/10.5852/ejt.2022.798.1679 


\section{Introduction}

Asplenium L. (Aspleniaceae) is an approximately globally distributed genus comprising over 700 extant species (Kramer \& Viane 1990). Reticulate evolution caused by hybridization and polyploidy has generated various species complexes that pose difficulty with species delimitation in the genus, e.g., the A. monanthes complex (Dyer et al. 2012), the A. normale complex (Chang et al. 2018), the $A$. pekinense complex, and the $A$. varians complex (Liang et al. 2021). The $A$. coenobiale complex belongs to $A$. ser. Variantia Ching \& S.H.Wu (Wu 1999). Members in this complex are characterized by the presence of small finely dissected leaves, and shiny castaneous to purplish black and threadlike stipes, and petioles.

Asplenium coenobiale complex is mainly distributed in the tropical and subtropical regions of southern and southwestern China, Malaysia, southern Japan, and northern Vietnam (Lin \& Viane 2013). Members within this complex are morphologically variable and species delimitation is difficult. Wu (1999) recognized four species in total within this complex, including A. coenobiale Hance, A. fuscipes Bak., A. subtoramanum Ching ex S.H.Wu, and A. toramanum Makino. Lin \& Viane (2013) treated the latter three species as synonyms of $A$. coenobiale and believed that this species has variable frond division probably depending on growing conditions with large and more divided plants often growing on rocks in forests. However, Lin \& Viane (2013) retained Asplenium pulcherrimum (Baker) Ching ex Tardieu, which had been confused with $A$. coenobiale, and the similar diploid species $A$. cornutissimum X.C.Zhang \& R.H.Jiang. Most recently, Liang et al. (2019) made an integrative taxonomic study using evidence of morphology, cytology, and molecular phylogeny to resolve the species delimitation of the complex. On the basis of treatment by Lin \& Viane (2013), Liang et al. (2019) described one autotetraploid novel species (A. maguanense S.Q.Liang, R.Wei \& X.C.Zhang) in addition to the species recognized by Lin \& Viane (2013).

However, the previous studies on the integrative taxonomy of the A. coenobiale complex mainly focused on samples from China's Guizhou, Guangxi, and Yunnan provinces, as well as a small number of representatives from China's Taiwan Island, Japan, and Vietnam (Liang et al. 2019; Xu et al. 2020). Their taxonomic analyses of this complex did not involve samples from South China's Guangdong province. By examinations of specimens from Danxia mountain of Guangdong province and the comparative analyses of the whole complex, we hypothesized that these samples from Danxia mountain, Guangdong, represented a cryptic new species. In the present study, we reconducted a taxonomic analysis by adding samples from Danxia mountain, Guangdong, which is one of the famous examples of Danxia landform characterized by red-colored sandstones and conglomerates of largely Cretaceous age (Peng 2009). We aimed to detect the presence of the new cryptic species of $A$. coenobiale complex in this special habitat of Danxia landform.

\section{Material and methods}

\section{Material and taxon sampling}

To detect the presence of cryptic species in Asplenium coenobiale complex in Danxia landform, we newly generated DNA sequence data including three plastid genes and one nuclear gene of the two samples of the complex from a cave of Danxia mountain, Guangdong province. We also included accessions of the A. coenobiale complex from the previous studies of Liang et al. (2019) and Xu et al. (2020). A total of five plastid genes of 53 accessions and one nuclear gene of 33 accessions as the ingroup were incorporated into the molecular analyses (Supplementary file 1). The selection of outgroup taxa reflected the phylogenetic relationships between the $A$. coenobiale complex and its closely related species (Xu et al. 2020).

\section{Spore observation and cytotype determination}

Scanning Electron Microscope (SEM) images were taken of the spores of members in the A. coenobiale complex. Spores obtained from herbarium specimens, were mounted on specimen tabs and then coated 
with platinum in a sputter coater. Observations were conducted using an ESEM-Quanta 200 (FEI, Hillsboro, Oregon, USA) with $15 \mathrm{Kv}$ at Nanjing Forestry University, Nanjing, China. The ImageJ software (Pérez \& Pascau 2013) was used for measurement on SEM micrographs. For plants of the A. coenobiale complex from Danxia mountain, DNA ploidy levels were determined by measuring DNA-C values using a BD FACScalibur, and Zea mays L. was employed as the internal standard. The obtained data were then compared to the previously recorded genome size of members in this complex (Liang et al. 2019).

\section{DNA extraction, PCR amplification, and sequencing}

Total genomic DNA was extracted from silica-gel-dried leaves using the modified $2 \times \mathrm{CTAB}$ procedure of Doyle \& Doyle (1987). Three plastid markers ( $r b c L$, rps 4 , and the rps4-trnS intergenic spacer) and one nuclear gene (pgiC) were selected for amplification and sequencing. The $r b c L$ gene was amplified with primers ESRBCL1F and ESRBCL1361R (Schuettpelz \& Pryer 2007). The rps4 gene and the rps4-trnS intergenic spacer were amplified using the forward primer 5'-ATG TCMCGTTAYCGAGGRCCTCGT-3' and the reverse primer 5'-TACCGAGGGTTCGAATC-3' (Schneider et al. 2005). The nuclear gene pgiC was amplified using primers 14F and 16R (Ishikawa et al. 2002). The PCR mixtures and cycling parameters of plastid genes followed those of Ohlsen et al. (2015). Amplified fragments were purified with TIANquick mini purification kits (TIANGEN, Beijing, China). Purified PCR products were sequenced by Sangon Biotech (Shanghai, China). For the nuclear gene, the purified nuclear DNA products were ligated into a pUCm-T Vector. Ten positive clones for each individual were randomly selected for sequencing.

\section{Sequence alignment and phylogenetic analysis}

The newly generated sequences were assembled and edited using Sequencher ver. 4.14 (GeneCodes Corporation, Ann Arbor, Michigan). Subsequently, all sequences were initially aligned with MAFFT ver. 7 (Katoh \& Standley 2013) and manually adjusted in BioEdit (Hall 1999). Alignments of six cpDNA regions were concatenated using PhyloSuite (Zhang et al. 2020). Independent phylogenetic analyses of the plastid and nuclear datasets were conducted using maximum likelihood (ML) and Bayesian inference (BI) on the Cipres web server (Miller et al. 2010), respectively. The ML tree searches were performed using RAxML-HPC2 on XSEDE with 1000 bootstrap replicates. The model TPM1uf + G for combined plastid DNA dataset and HKY for nuclear dataset in maximum likelihood analyses was used based on results of jModelTest2 (Darriba et al. 2012) on XSEDE (2.1.6) using the Bayesian information criterion (BIC). The BI was conducted using MrBayes ver. 3.2.7a (Ronquist \& Huelsenbeck 2003) with temperature parameter set to 0.2 , and other priors set to their default values. Two independent runs, each with four chains (one cold, three heated), were conducted, each beginning with a random tree and sampling one tree every 1000 generations of 10000000 generations. Convergence among runs and stationarity were assessed using Tracer ver. 1.4 (Rambaut \& Drummond 2007), and a burnin was discarded. The remaining trees were used to calculate a $50 \%$ majority-rule consensus topology and posterior probabilities $(\mathrm{PP})$.

\section{Results}

\section{Molecular analysis}

The 64 aligned cpDNA sequences are 5105 bp in length, with 525 parsimony informative sites in total and the 78 aligned nuclear sequences are $717 \mathrm{bp}$ in length, with 20 parsimony informative sites. The present result from the combined plastid dataset shows that the two samples of Asplenium from Danxia mountain form an independent clade that is clearly separated from all the other species in the phylogenetic tree (Fig. 1), while the nuclear dataset resolved the three pgiC alleles of collection XKW674 into two well-supported clades (Fig. 2). 


\section{Taxonomic treatment}

Class Polypodiopsida Cronquist, Takht. \& W.Zimm.

Subclass Polypodiidae Cronquist, Takht. \& W.Zimm.

Order Polypodiales Link

Suborder Aspleniineae H.Schneid. \& C.J.Rothf.

Family Aspleniaceae Newman

Genus Asplenium L.

Asplenium danxiaense K.W.Xu sp. nov.

urn:lsid:ipni.org:names:77260762-1

Figs 3A, E, 4, 5A-B

\section{Diagnosis}

Asplenium danxiaense K.W.Xu sp. nov. somewhat resembles $A$. coenobiale and A. pulcherrimum by its dark brown to black rhizome scale with fimbriate to subentire margin, shiny dark brown to black,

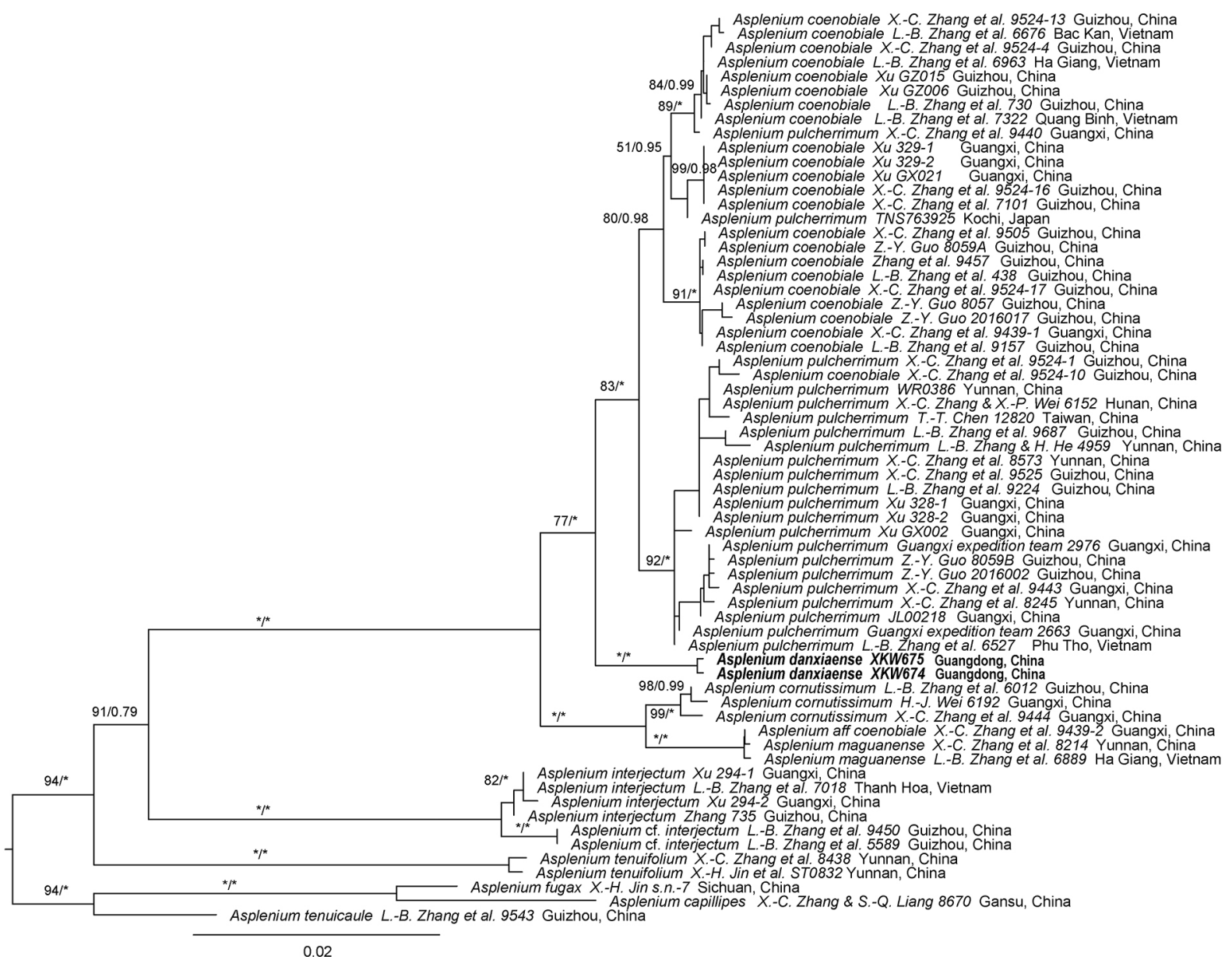

Fig. 1. The phylogenetic position of Asplenium danxiaense K.W.Xu sp. nov. based on five plastid markers (atpB, rbcL, rps4-trnS, rpl32-trnP, and trnL-F). The numbers associated with branches are maximum likelihood bootstrap (MLBS) values followed by bayesian inference posterior probabilities (PP). * indicates MLBS $=100 \%$ or $\mathrm{PP}=1$. 
rigid and threadlike stipe and rachis, finely dissected frond, and lophate (cristate-alate) perispore, but the former has rhizome scales narrowly triangular to lanceolate, with a short apical tail, basal-most basiscopic secondary pinnae usually largest, fertile segment scarce, and exospore length usually more than $50 \mu \mathrm{m}$. In contrast, $A$. coenobiale and A. pulcherrimum have scales narrowly triangular to linearsubulate, with a long apical tail, basal acroscopic pinnule usually largest, fertile segment abundant, and exospore length usually less than $50 \mu \mathrm{m}$.

\section{Etymology}

Based on the mountain name, Danxia, in northern Guangdong, China, and the Latin suffix, -ense, of origin, referring to the type locality of the species.

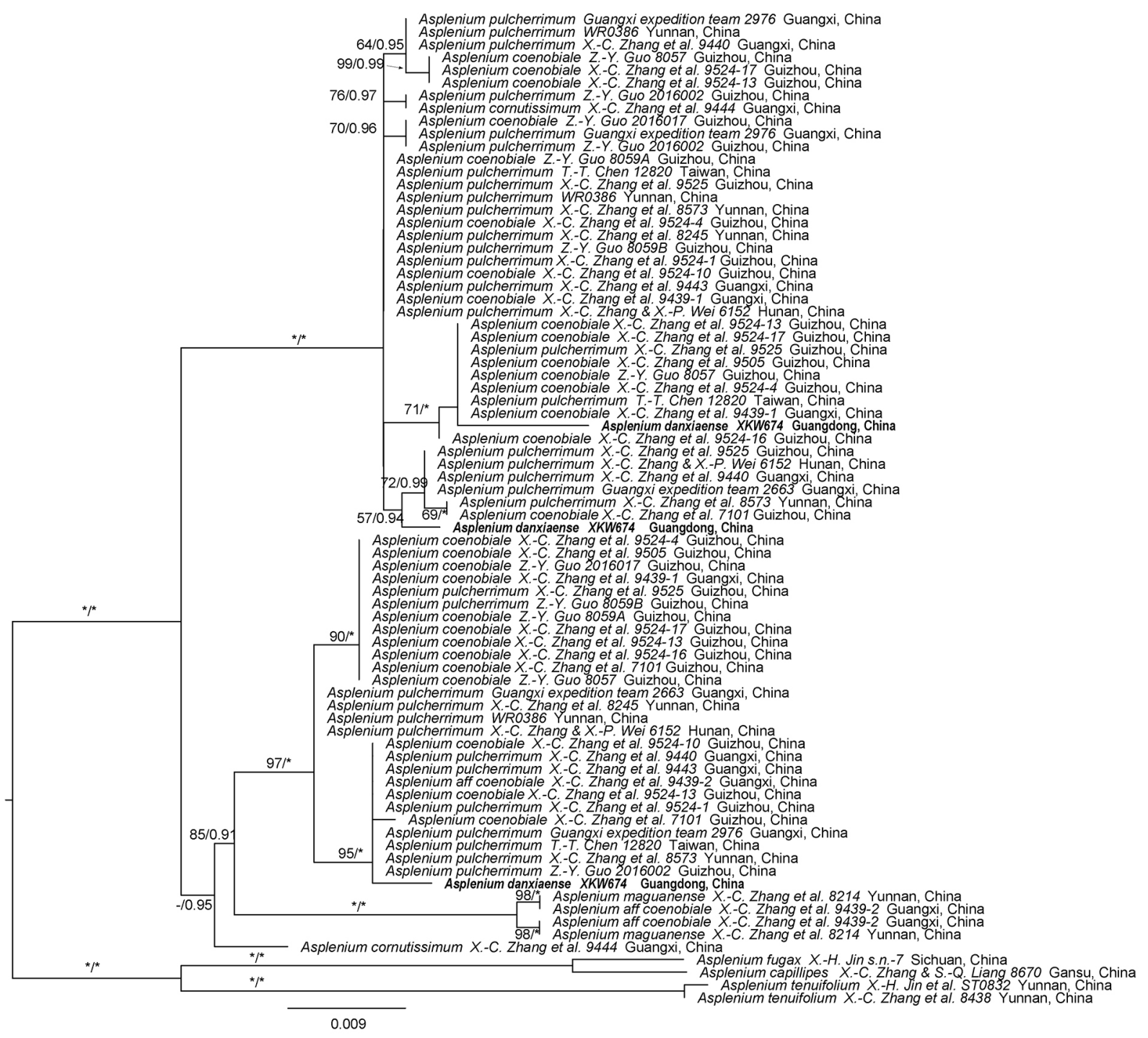

Fig. 2. The phylogenetic position of Asplenium danxiaense sp. nov. based on nuclear gene pgiC. The numbers associated with branches are maximum likelihood bootstrap (MLBS) values followed by bayesian inference posterior probabilities (PP). * indicates MLBS $=100 \%$ or $\mathrm{PP}=1$. 


\section{Type material}

\section{Type}

CHINA - Guangdong province, Shaoguan City, Renhua County, Danxia mountain; 2501'41.076" N, $113^{\circ} 41^{\prime} 44.982^{\prime \prime} \mathrm{E}$; elev. $150 \mathrm{~m}$; in evergreen broad-leaved forest in a cave of Danxia landform; 12 May 2021; Jian-Qiang Guo \& Xin-Xin Zhou XKW674; holotype: NF!; isotype: SYS!.

\section{Description}

Plants up to $30 \mathrm{~cm}$ tall. Rhizome erect, apex scaly; scales dark brown to black, narrowly triangular to lanceolate, $(2.5-) 4-5 \times 0.4-0.9 \mathrm{~cm}$, clathrate, margin fimbriate to subentire, apex shortly hairpointed. Fronds caespitose; stipe shiny, purplish black, 10-16(-20) cm, terete, rigid and threadlike, subglabrous; rachis shiny purplish black, becoming green in upper half toward apex, sulcate adaxially, subglabrous; lamina firmly herbaceous, green, subglabrous, triangular to ovate-triangular, 8-12 $\times$ 4-7 cm, base truncate, (2 or) 3-pinnate, apex acuminate; primary pinnae in (6-) 8-14 pairs, subopposite to alternate, overlapping, stalk short, purplish black to green abaxially, basal pinnae largest, ovatelanceolate, 3-6 × 1.5-2 cm, base obliquely truncate to truncate, apex subacute; secondary pinnae in 3-7 pairs, anadromous, basal basiscopic secondary pinnae usually largest, triangular-ovate, $0.7-1.5 \times$ 0.5-0.8 cm, stalk short to subsessile, base asymmetrical, acroscopic side truncate, basiscopic side cuneate, apex obtuse; ultimate segments $2-4$ pairs, ovate-oblong to linear, apex with 2-4 short and broadly triangular, obtuse to submucronate or sharp teeth. Costa and costules sulcate adaxially, green, veins slightly raised or flat adaxially, anadromous, 1 vein per segment, not reaching margin. Sori 1 per

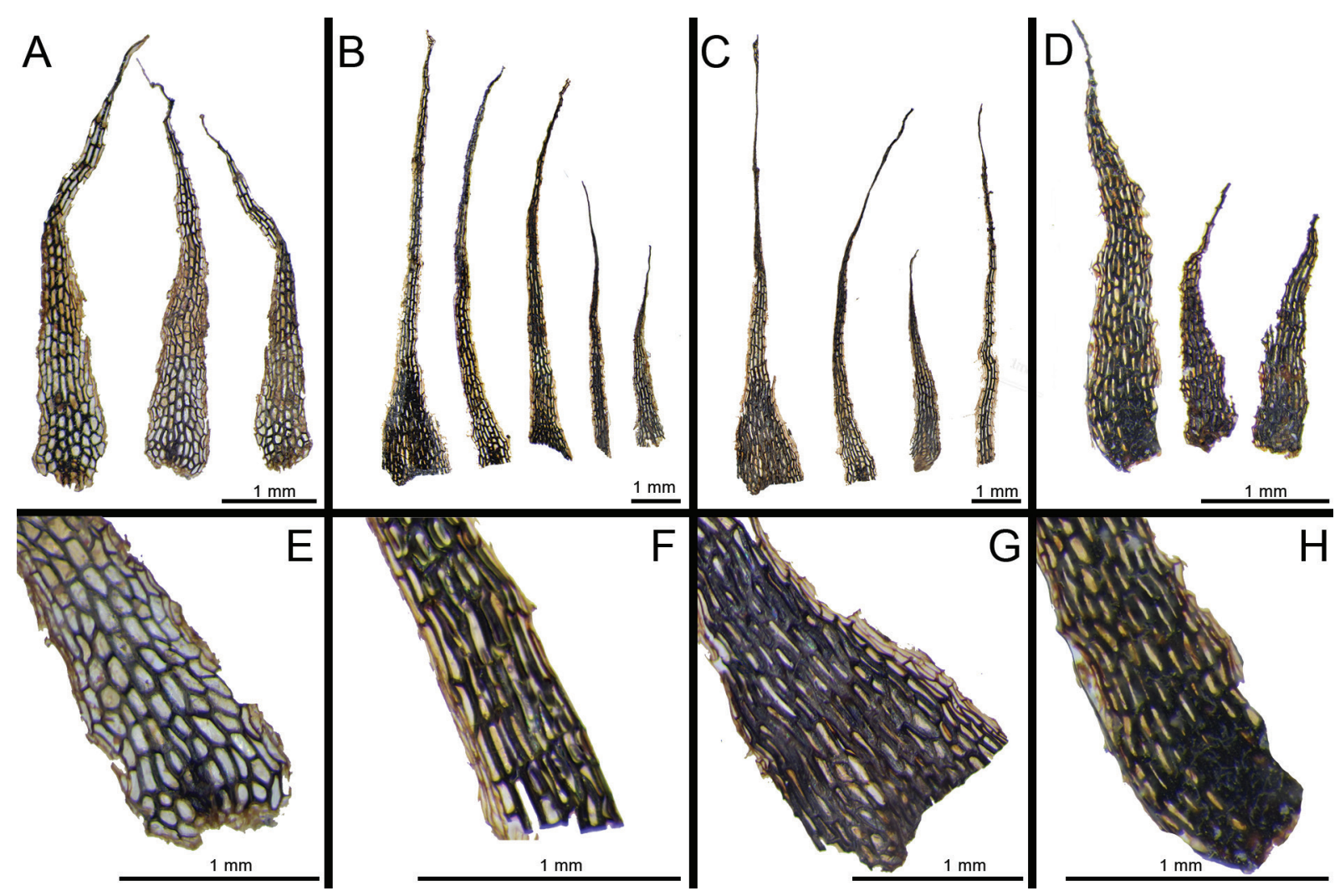

Fig. 3. Scale morphology of the new species and its affinities. A, E. Asplenium danxiaense K.W.Xu sp. nov. B, F. A. pulcherrimum (Baker) Ching ex Tardieu. C, G. A. coenobiale Hance. D, H. A. cornutissimum X.C.Zhang \& R.H.Jiang. 
fertile, forked and pouch-shaped segment, medial to subterminal on acroscopic veinlet, oval to linear, 1-3 mm long; indusium grayish green, oval to linear, membranous, margin nearly entire, persistent, opening toward costules, persistent. Spores brown to dark brown, exospore 52-60 $\mu \mathrm{m}$ long, lophate (cristate-alate) perispore.
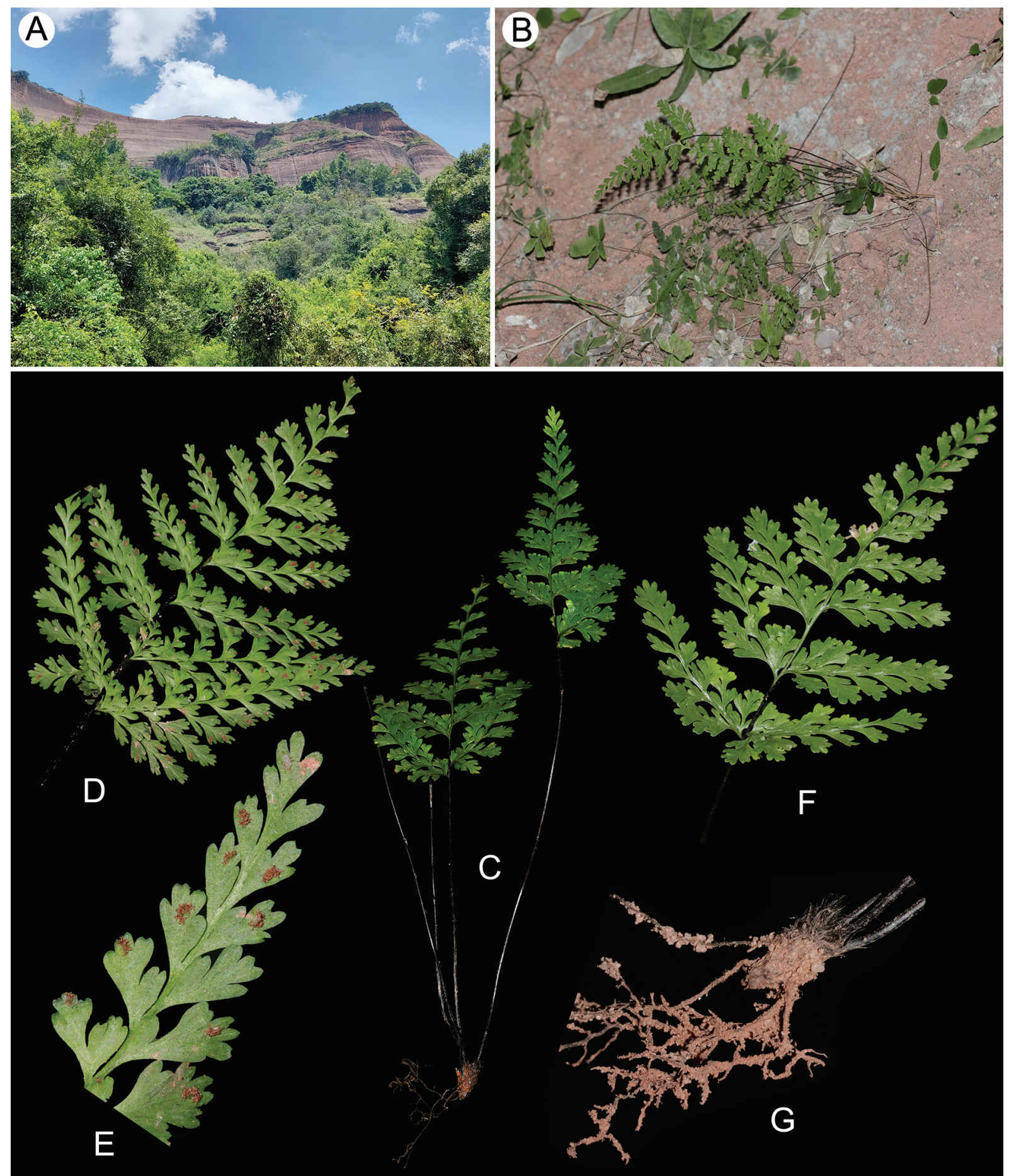

Fig. 4. Asplenium danxiaense K.W.Xu sp. nov. A. Danxia landform in the type locality of the new species. B. Habitat of the new species in a cave. C. Habit. D. Abaxial view of lamina. E. Abaxial view of lamina apex. F. Adaxial view of lamina. E. Rhizome and root. 


\section{Additional material}

CHINA - Guangdong province, Shaoguan City, Renhua County, Danxia mountain; 2501'41.076" N, $113^{\circ} 41^{\prime} 44.982^{\prime \prime} \mathrm{E}$; elev. $150 \mathrm{~m}$; in evergreen broad-leaved forest in a cave of Danxia landform; 12 May 2021; Jian-Qiang Guo \& Xin-Xin Zhou XKW675; NF!.

\section{Distribution and habitat}

Asplenium danxiaense sp. nov. is only known from Guangdong, China, growing in a cave of Danxia mountain in evergreen broad-leaved forest, at the elevation of 100-300 m.

\section{Vernacular name}

We propose a Chinese name, Dānxiáshān tiějiăojué (丹霞山铁角菜) to reflect the type locality of the new species.

\section{Conservation Status}

We provisionally assess Asplenium danxiaense sp. nov. as Critically Endangered based on criterion D of IUCN (2012). Only one population and fewer than 50 individuals of $A$. danxiaense sp. nov. are known from Danxia mountain. It was not seen by one of us during separate expeditions to nearby sites on Danxia mountain.

\section{Discussion}

Members of the Asplenium coenobiale complex are mainly distributed in karst mountains of south and southwestern China's Guangxi, Guizhou, and Yunnan provinces, also extending to adjacent regions

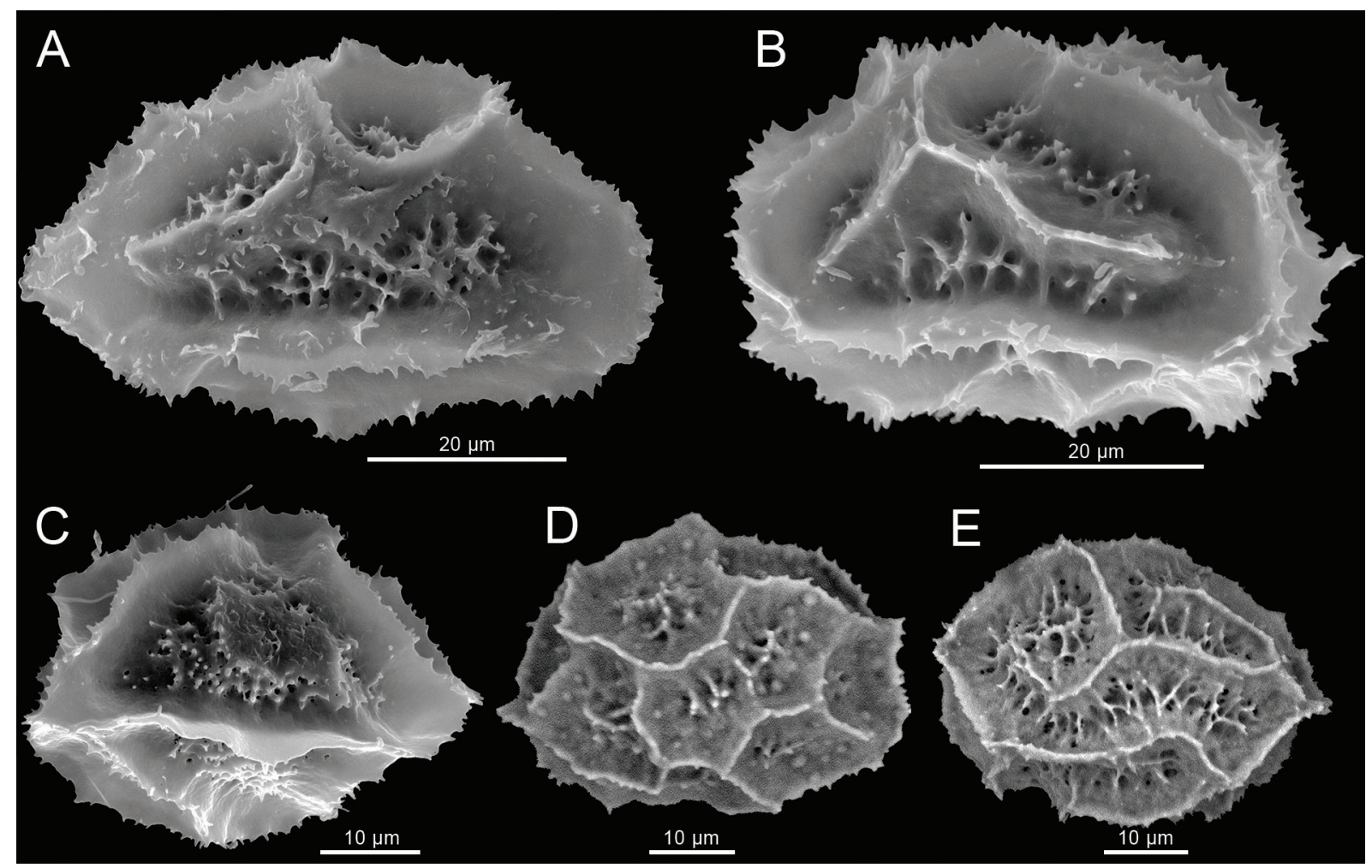

Fig. 5. Spores of the new species Asplenium danxiaense K.W.Xu sp. nov. and its affinities. A, B. A. danxiaense K.W.Xu sp. nov. C. A. cornutissimum X.C.Zhang \& R.H.Jiang. D. A. coenobiale Hance. E. A. pulcherrimum.(Baker) Ching ex Tardieu. 
including Guangdong, Hunan, and Taiwan Island of China, Japan, and Vietnam (Lin \& Viane 2013). Four species within the complex have been recognized by recent study (Liang et al. 2019). During our field investigation in Danxia mountain of Guangdong province, we found some plants of the $A$. coenobiale complex in a cave. These plants are morphologically close to A. coenobiale and A. pulcherrimum, but they could be obviously distinguished based on the morphological and micromorphological characters. The rhizome scales of the plants from Danxia mountain are narrowly triangular to lanceolate, with a short apical tail, while those of $A$. coenobiale and A. pulcherrimum are narrowly triangular to linear-subulate, with a long apical tail (Fig. 3). In the plants from Danxia mountain, the basal-most basiscopic secondary pinnae are larger than the basal-most acroscopic secondary pinnae (Fig. 4), while in A. coenobiale and $A$. pulcherrimum, the basal-most acroscopic secondary pinnae are usually larger than the basalmost basiscopic secondary pinnae. In addition, plants from Danxia mountain usually have less fertile segments per frond than those of $A$. coenobiale and A. pulcherrimum. The spore ornamentation of plants from Danxia mountain is lophate (cristate-alate) perispore, which is similar to that of $A$. coenobiale and A. pulcherrimum (Fig. 5), while the exospore length of plants from Danxia mountain (usually more than

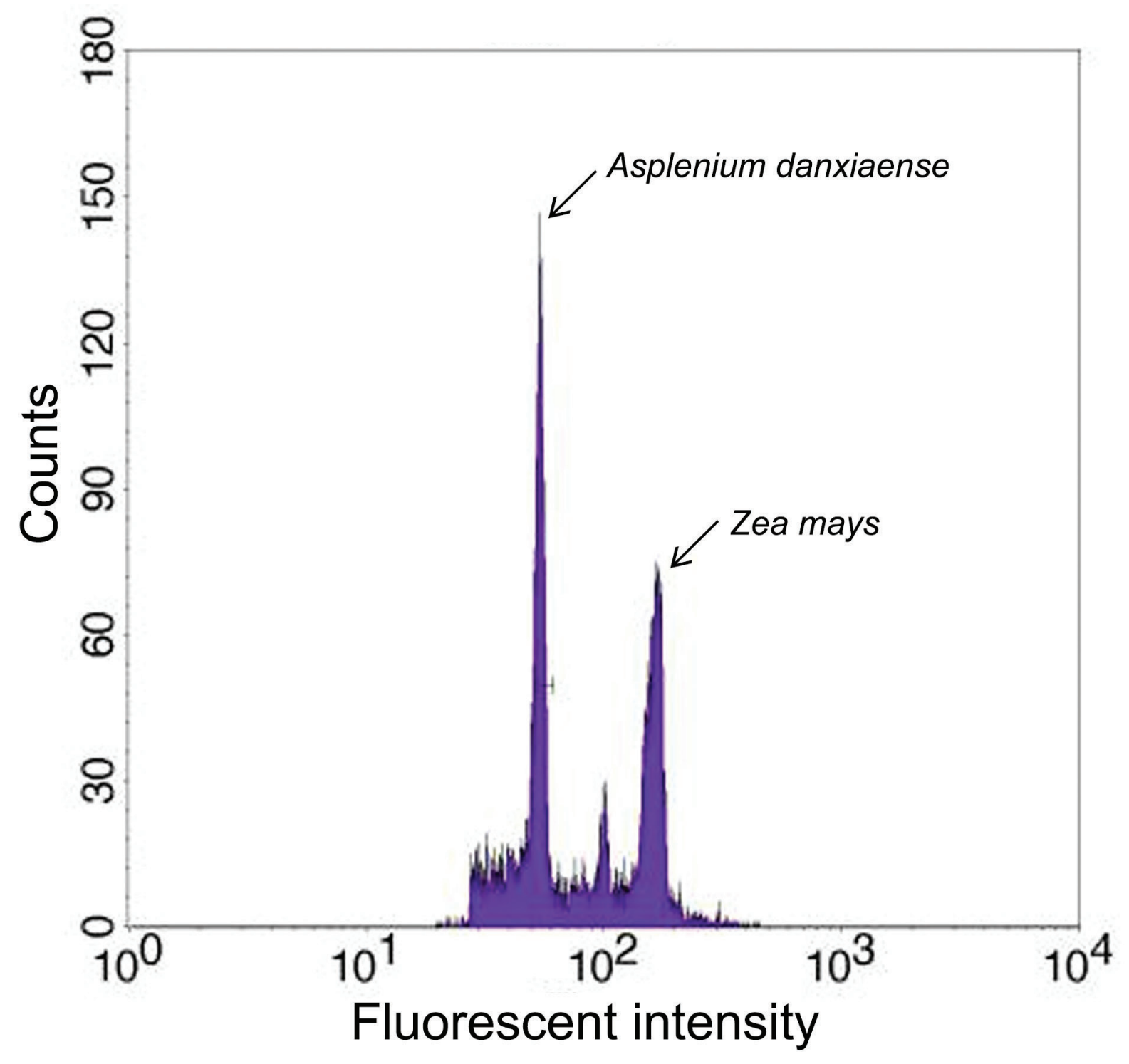

Fig. 6. Estimation of Asplenium danxiaense K.W.Xu sp. nov. genome size by flow cytometry. The internal control Zea mays L. cv. B73 has $1 \mathrm{C}=2.3 \mathrm{Gbp}$. 
$50 \mu \mathrm{m}$ ) is longer than the exospore length of species A. coenobiale and A. pulcherrimum (usually less than $50 \mu \mathrm{m}$ ). Thus, a new species, $A$. danxiaense K.W.Xu, sp. nov., is proposed here. This new species also resembles species $A$. cornutissimum and A. maguanense. However, it differs from A. cornutissimum by its plants more strong, tetraploid, laminae firmly herbaceous, widest at base, and exospore length usually more than $50 \mu \mathrm{m}$, and differs from A. maguanense by its laminae 3-pinnate, ultimate segment apex with long teeth, and exospore length usually more than $50 \mu \mathrm{m}$ (Jiang et al. 2011; Liang et al. 2019).

The genome size of the new species $A$. danxiaense sp. nov. is estimated as $1 \mathrm{C}=7.06 \mathrm{~Gb}$, namely $14.8 \mathrm{pg} / 2 \mathrm{C}$ ( $1 \mathrm{pg} \approx 978 \mathrm{Mb}$; Dolezel et al. 2003), which is close to the value previously estimated for tetraploid taxa (A. coenobiale, A. maguanense and A. pulcherrimum) in the A. coenobiale complex (Fig. 6; Lin \& Viane 2013; Liang et al. 2019). Moreover, the spore size of the new species is somewhat larger than that of other tetraploid taxa in the A. coenobiale complex. Based on the genome size and spore size, we speculated that the new species from Danxia mountain is also a tetraploid species.

Phylogenetically, the new species was well resolved as a distinct clade, which is sister to a clade containing accessions of $A$. coenobiale and $A$. pulcherrimum based on phylogeny reconstructed using maternally inherited plastid genes (Fig. 1). In contrast, a close relationship between A. danxiaense, A. coenobiale and A. pulcherrimum was also supported by their sharing of similar gene copies in the nuclear phylogeny (Fig. 2). The three $p g i C$ alleles present in $A$. danxiaense sp. nov., are nested within two well-supported clades (Fig. 2). Plastid genes confidently resolved relationships of the $A$. coenobiale complex into five major clades corresponding to five species recognized in this complex, while the nuclear pgiC gene resolved members of this complex into four major clades. Conflicts between the plastid and low-copy nuclear phylogenies at species level were clearly observed in this complex. This discordance might be caused by reticulation events involving hybridization and allopolyploidization, as has been frequently documented in Asplenium (Dyer et al. 2012; Fujiwara et al. 2017; Chang et al. 2018; Liang et al. 2019, 2021).

\section{Acknowledgments}

We are grateful to Liang Zhang for helping with the flow cytometry. This work was supported by the National Natural Science Foundation of China (\#32100167), the Natural Science Foundation of Jiangsu Province (\#BK20210612), the Nanjing Forestry University project funding (\#163108093), the Guangzhou Science and Technology Program (\#201903010076), and the Strategic Priority Research Program of the Chinese Academy of Sciences (\#XDB31000000). We thank the editor and the other anonymous reviewers for helpful comments.

\section{References}

Chang Y.-F., Ebihara A., Lu S.-G., Liu H.-M. \& Schneider H. 2018. Integrated taxonomy of the Asplenium normale complex (Aspleniaceae) in China and adjacent areas. Journal of Plant Research 131: 573-587. https://doi.org/10.1007/s10265-018-1032-y

Darriba D., Taboada G. L., Doallo R. \& Posada D. 2012. jModelTest 2: more models, new heuristics and parallel computing. Nature Methods 9 (8): 772-772. https://doi.org/10.1038/nmeth.2109

Dolezel J., Bartos J., Voglmayr H. \& Greilhuber J. 2003. Nuclear DNA content and genome size of trout and human. Cytometry. Part A: the journal of the International Society for Analytical Cytology 51 (2): 127-128. https://doi.org/10.1002/cyto.a.10013

Doyle J.J. \& Doyle J.L. 1987. A rapid DNA isolation procedure for small quantities of fresh leaf tissue. Phytochemical Bulletin 19: 11-15.

Dyer R.J., Savolainen V. \& Schneider H. 2012. Apomixis and reticulate evolution in the Asplenium monanthes fern complex. Annals of Botany 110: 1515-1529. https://doi.org/10.1093/aob/mes202 
Fujiwara T., Uehara A., Iwashina T., Matsumoto A., Chang Y.H., Chao Y.S. \& Watano Y. 2017. Allotetraploid cryptic species in Asplenium normale in the Japanese Archipelago, detected by chemotaxonomic and multi-locus genotype approaches. American Journal of Botany 104: 1390-1407. https://doi.org/10.3732/ajb.1700141

Hall T.A. 1999. BioEdit: a user-friendly biological sequence alignment editor and analysis program for Windows 95/98/NT. Nucleic Acids Symposium Series 41: 95-98.

IshikawaH., Watano Y., KanoK., Ito M.\& Kurita S. 2002. Development of primersetsfor PCRamplification of the pgiC gene in ferns. Journal of Plant Research 115: 65-70. https://doi.org/10.1007/s102650200010

IUCN. 2012. IUCN Red List Categories and Criteria: Version 3.1. Second edition. Gland, Switzerland. Available from https://portals.iucn.org/library/node/10315 [accessed 5 November 2021].

Jiang R.-H., Zhang X.-C. \& Liu Y. 2011. Asplenium cornutissimum (Aspleniaceae), a new species from karst caves in Guangxi, China. Brittonia 63 (1): 83-86. https://doi.org/10.1007/s12228-010-9139-z

Katoh K. \& Standley D.M. 2013. MAFFT multiple sequence alignment software version 7: improvements in performance and usability. Molecular Biology and Evolution 30: 772-780.

https://doi.org/10.1093/molbev/mst010

Kramer K.U. \& Viane R. 1990. Aspleniaceae. In: Kubitzki K., Kramer K.U. \& Green P.S. (eds) The Families and Genera of Vascular Plants. Vol. 1. Pteridophytes and Gymnosperms: 52-57. Springer, Berlin. https://doi.org/10.1007/978-3-662-02604-5_14

Liang S.-Q., Zhang X.-C. \& Wei R. 2019. Integrative taxonomy resolved species delimitation in a fern complex: A case study of the Asplenium coenobiale complex. Biodiversity Science 27 (11): 1205-1220. https://doi.org/10.17520/biods.2019316

Liang S.-Q., Viane R.L.L., Zhang X.-C. \& Wei R. 2021. Exploring the reticulate evolution in the Asplenium pekinense complex and the A. varians complex (Aspleniaceae). Journal of Systematics and Evolution 59 (1): 125-140. https://doi.org/10.1111/jse.12530

Lin Y.-X. \& Viane R.L.L. 2013. Aspleniaceae. In: Wu Z.-Y., Raven P.H. \& Hong D.-Y. (eds) Flora of China. Vol. 2-3: 267-316. Science Press/Missouri Botanical Garden Press, Beijing and St. Louis.

Miller M.A., Pfeiffer W. \& Schwartz, T. 2010. Creating the CIPRES Science Gateway for inference of large phylogenetic trees. In: Proceedings of the Gateway Computing Environments Workshop (GCE): 1-8. 14 Nov 2010, New Orleans, LA. https://doi.org/10.1109/GCE.2010.5676129

Ohlsen D.J., Perrie L.R., Shepherd L.D., Brownsey P.J. \& Bayly M.J. 2015. Phylogeny of the fern family Aspleniaceae in Australasia and the southwestern Pacific. Australian Systematic Botany 27: 355371. https://doi.org/10.1071/SB14043

Peng H. 2009. The concept, research history and existing problems of Danxia. In: Peng H. (ed.) First international symposium on Danxia landform. Mount Danxia, Shaoguan, Guangdong, China.

Pérez J.M.M. \& Pascau J. 2013. Image processing with ImageJ. Packt Publishing Ltd., Birmingham.

Rambaut A. \& Drummond A.J. 2007. Tracer 1.4. Available from http://beast.bio.ed.ac.uk/Tracer [accessed 15 Oct. 2021].

Ronquist F. \& Huelsenbeck J.P. 2003. MrBayes 3: Bayesian phylogenetic inference under mixed mixed models. Bioinformatics 19: 1572-1574. https://doi.org/10.1093/bioinformatics/btg180

Schneider H., Ranker T.A., Russell S.J., Cranfill R., Geiger J.M., Aguraiuja R., Wood K.R., Grundmann M., Kloberdanz K. \& Vogel J.C. 2005. Origin of the endemic fern genus Diella coincides with the renewal of Hawaiian terrestrial life in the Miocene. Proceedings of the Royal Society B: Biological Sciences 272: 455-460. https://doi.org/10.1098/rspb.2004.2965 
Schuettpelz E. \& Pryer K.M. 2007. Fern phylogeny inferred from 400 leptosporangiate species and three plastid genes. Taxon 56: 1037-1050. https://doi.org/10.2307/25065903

Wu S.-H. 1999. Aspleniaceae. In: Wu Z.-Y. (ed.) Flora Reipublicae Popularis Sinicae. Vol. 6: 3-127. Science Press, Beijing.

Xu K.-W., Zhang L., Rothfels C.J., Smith A.R., Viane R.L.L., Lorence D., Wood K.R., Chen C.W., Knapp R., Zhou L., Lu N.T., Zhou X.-M., Wei H.-J., Fan Q., Chen S.-F., Cicuzza D., Gao X.-F., Liao W.-B. \& Zhang L.-B. 2020. A global plastid phylogeny of the fern genus Asplenium (Aspleniaceae). Cladistics 36 (1): 22-71. https://doi.org/10.1111/cla.12384

Zhang D., Gao F., Jakovlić I., Zou H., Zhang J., Li W.-X. \& Wang G.-T. 2020. PhyloSuite: an integrated and scalable desktop platform for streamlined molecular sequence data management and evolutionary phylogenetics studies. Molecular Ecology Resources 20 (1): 348-355.

https://doi.org/10.1111/1755-0998.13096

Manuscript received: 17 November 2021

Manuscript accepted: 7 January 2022

Published on: 7 March 2022

Topic editor: Frederik Leliaert

Desk editor: Fariza Sissi

Printed versions of all papers are also deposited in the libraries of the institutes that are members of the EJT consortium: Muséum national d'histoire naturelle, Paris, France; Meise Botanic Garden, Belgium; Royal Museum for Central Africa, Tervuren, Belgium; Royal Belgian Institute of Natural Sciences, Brussels, Belgium; Natural History Museum of Denmark, Copenhagen, Denmark; Naturalis Biodiversity Center, Leiden, the Netherlands; Museo Nacional de Ciencias Naturales-CSIC, Madrid, Spain; Real Jardín Botánico de Madrid CSIC, Spain; Zoological Research Museum Alexander Koenig, Bonn, Germany; National Museum, Prague, Czech Republic.

\section{Supplementary file}

Supp. file 1. List of voucher specimens and Genbank accession numbers used in phylogenetic analyses. - indicates missing data. https://doi.org/10.5852/ejt.2022.798.1679.6143 